\title{
Orbital period modulation and spot activity in the RS CVn binary V711 Tauri ${ }^{\star}$
}

\author{
S. Muneer ${ }^{1}$, K. Jayakumar ${ }^{2}$, M. J. Rosario ${ }^{2}$, A. V. Raveendran ${ }^{1}$, and M. V. Mekkaden ${ }^{1}$ \\ 1 Indian Institute of Astrophysics, Bangalore 560034, India \\ e-mail: [muneers;mvm;avr]@iiap.res.in \\ 2 Vainu Bappu Observatory, Indian Institute of Astrophysics, Kavalur 635701, India \\ e-mail: [mjr; jayakumar]@iiap.res.in
}

Received 4 May 2010 / Accepted 30 June 2010

\begin{abstract}
Aims. We attempt to establish the real nature of the orbital period variation and its relation to the spot activity of V711 Tau, and determine why the $(B-V)$ colour of the star appears to be nearly independent of its $V$ magnitude. We wish to verify whether existing predictions in the literature for the long-term spot activity of the star are true or not by making extended photometric observations, and whether the broad component of $\mathrm{H} \alpha$ emission originates in more localised active regions, as suggested by some chromospheric models, by searching for any correlation between the base-line width of the emission and the light modulation.

Methods. We obtained new radial velocities of the G-type component of V711 Tau on 42 nights during 2004-09, and $B V$ photometry of V711 Tau on 202 nights during the years 1993-2009. We measured the equivalent widths of two well resolved lines of the G-type component from 13 of the spectra obtained by us. We also measured the equivalent widths and base-line widths of $\mathrm{H} \alpha$ emission from 21 spectra obtained during 2008-09. We analyse these along with relevant information available in the literature.

Results. The available radial velocity data of V 711 Tau are consistent with a sinusoidal modulation of its orbital period; the period of modulation is found to be $36.3 \pm 1.9 \mathrm{yr}$. The fractional light-loss over a photometric cycle is found to vary with an average period of $14.1 \pm 0.3 \mathrm{yr}$. It appears that physical processes linked to the magnetic activity of the spotted star are responsible for the variation in the orbital period. The excess reduction in the $B$ band flux relative to that in $V$ band caused by spot activity is almost compensated for by the fractional increase in the contribution by the hotter companion to the total light in the blue spectral region, and thereby makes the $(B-V)$ colour of the binary system nearly independent of its $V$ magnitude. We find that the ratio of the radii of the components derived from their $v \sin i$ values, which is usually quoted in the literature, is substantially larger than that implied by the ratio of their brightnesses in $V$ band. There is a slight indication from the $V$ band data that the spot activity in V711 Tau has a component that is fixed in the orbital frame of reference. The equivalent width and base-line width of $\mathrm{H} \alpha$ emission do not show any obvious correlation with the $V$ band light curve.
\end{abstract}

Key words. stars: activity - binaries: spectroscopic - stars: individual: V711 Tau - stars: late-type - starspots

\section{Introduction}

V711 Tau (HR 1099) is one of the bright members of the RS Canum Venaticorum binaries, and it has been the object of several photometric and spectroscopic studies since the discovery of its light variability in 1975 by Landis \& Hall (1976). It is a non-eclipsing, double-lined spectroscopic binary with a $\mathrm{K} 0-\mathrm{K} 1$ subgiant as primary and a G5 dwarf as secondary in an almost circular orbit (Fekel 1983).

The variation in the orbital period of V711 Tau was wellestablished by Frasca \& Lanza (2005), who found the difference between the observed and computed times of maximum of the radial velocity of the K-type primary, $\mathrm{O}-\mathrm{C}$, to be constant from about 1976 to 1990 and to decrease steeply thereafter. They fitted a fourth order polynomial to the $\mathrm{O}-\mathrm{C}$ data available up until 2002 to derive the variation in $\Delta P / P$ over time. Their analysis shows that the $\Delta P / P$, probably, has a periodic component of amplitude about $\pm 5 \times 10^{-5}$ superimposed on a constant offset around $-3 \times 10^{-5}$. The constant offset implies that the period

* Full Table 2 is only available in electronic form at the CDS via anonymous ftp to cdsarc.u-strasbg.fr (130.79.128.5) or via http://cdsarc.u-strasbg.fr/viz-bin/qcat?J/A+A/521/A36 used in the computation of $\mathrm{O}-\mathrm{C}$ is around $8 \times 10^{-4}$ day longer than the actual period. Lanza et al. (2006), who later analysed the long-term spot evolution of V711 Tau, prefer the alternative interpretation that the orbital period was nearly constant up until about 1990, and following a sudden burst in the spot activity around that time, it subsequently started decreasing.

The analysis of the eclipse-times of Algol binaries by Hall (1990) demonstrated that cyclic variations in orbital periods are observed only in systems with a late-type component, suggesting that the orbital period modulations in these binaries may be related to the magnetic activities of the late-type components. Based on a study of the eclipse-times of a few RS CVn binaries that have simultaneous information about the spot area on the active component, Lanza \& Rodono (2004) found that the lengths of the orbital period and spot activity cycles are, probably, in the ratio 2:1. A clear picture of the nature of orbital period variation and its relationship with the photometric variation is essential in understanding the basic physical mechanism that produces the cyclic variation in the orbital periods of chromospherically active binaries.

V711 Tau is one of the four RS CVn binaries known to display always the $\mathrm{H} \alpha$ line as pure emission (Bopp 1983; 
Wacker et al. 1986). Fraquelli (1982) argued that both the primary and secondary contribute to the emission in the ratio $2.3: 1$, while Nations \& Ramsey (1986) concluded that the ratio is, probably, 6.1:1. Montes et al. (1997), however, attributed almost the entire emission to the K-type primary. They analysed the spectra of 18 chromospherically active stars in that study and found that the most active systems among them show very broad $\mathrm{H} \alpha$ emission wings and that the $\mathrm{H} \alpha$ emission features in these systems can be matched well by using a two-component Gaussian fit. In the case of V711 Tau, which was observed only on three consecutive nights, they found that a major fraction of the emission equivalent width is produced by the broad component, and that the contribution of the broad component, probably, has an orbital modulation. Based on a modelling of the chromosphere of the K-type primary using a two-component nonLTE model, Lanzafame et al. (2000) suggested that the broad emission component originates in more localised active regions. If this is true then the broad emission component should be well correlated with the photometric variation.

Lanza et al. (2006) modelled the $V$ band light curves of V711 Tau available up until 2002, while Berdyugina \& Henry (2007) modelled those available up until 2006 using inversion techniques. Their results imply that there are multi-periodicities in both the spot area and amplitude of light variation. Since the variations are not strictly periodic, to understand clearly the various timescales involved, it is imperative that the star is observed every season photometrically without any break.

It is generally understood that cool spots are responsible for the observed light modulation seen in chromospherically active stars, in which case the RS CVn stars should appear to be redder when fainter. However, in the case of V711 Tau the $(B-V)$ colour appears to be either nearly constant or becoming slightly bluer at fainter visual magnitudes. Ulvas \& Henry (2005), who modelled the $(B-V)$ colour variation in V711 Tau, found the fractional increase in the $B$-band flux of the hotter star to be insufficient to produce the observed peculiar $(B-V)$ variation, hence, proposed the presence of bright photospheric faculae along with the cool spots on the active star. In the case of the double-lined RS CVn binary UX Ari, which becomes appreciably bluer when fainter, Ulvas \& Engvold (2003) also suggested a similar mechanism. However, Rosario et al. (2007) pointed out that the unreasonably high brightness ratio of the components of UX Ari used by them in their modelling affected this conclusion, and that the greater contribution by the hotter secondary to the total light in the blue spectral region is definitely responsible for the star becoming bluer when it is fainter.

The most important parameter in modelling the $(B-V)$ colour variation in double-lined binaries is the relative brightnesses of the components in the $V$ spectral region when the active star is at its maximum brightness, corresponding to its unspotted phase. The difference in $V$ magnitudes of the components of V711 Tau when the active star is unspotted, $V_{K}-V_{G}$, used by Lanza et al. (2006) to model the $V$ band light curve is -1.4 , while that used by Ulvas \& Henry (2005) to model the $(B-V)$ variation is around -1.6 . Donati et al. (1992) used a value of -0.8 mag for the mean $V_{K}-V_{G}$ to compute the model $V$ light curves of V711 Tau from its temperature images obtained at two epochs during 1989-91. The mean $V$ magnitudes of the star at these epochs were $0.10-0.15$ below its maximum brightness ever observed, making the difference in the $V$ magnitudes of the components at the unspotted phase close to -1 . These values of $V_{K}-V_{G}$ are inconsistent with the difference in $B$ magnitudes, $B_{K}-B_{G}$, of -0.2 to 0.0 , suggested by Popper (1978) and Fekel (1983).
The last set of radial velocity observations available in the literature is that obtained at the epoch 2001.99 by Donati et al. (2003). We included V711 Tau in our spectroscopic programme in 2008 to systematically monitor the variations in its orbital period and $\mathrm{H} \alpha$ emission; the object has been in our photometric programme almost continuously since 1979 , apart from a break during 1995-2000. We had observed the star spectroscopically on several occasions earlier starting from 2004.

In this paper, we present new radial velocities of V711 Tau obtained during 2004-09, and its $B V$ photometry obtained during 1993-2009. We analyse the orbital period modulation of the binary system and its connection with the long-term spot activity of the cool component, and in the light of the results we discuss the mechanisms suggested to explain, in general, the orbital period variation in RS CVn binaries. The observed $(B-V)$ colour variation of V711 Tau with its brightness, which is unusual for an active star with cool spots on its surface, is modelled using a reasonable estimate of the relative brightnesses of the components from the observed equivalent widths of the absorption lines of the hotter component. We also analyse the $\mathrm{H} \alpha$ emission profiles obtained by us to search for any correlation with the photospheric light modulation.

\section{Observations}

During 2004-09, V711 Tau was observed on 42 nights with the fibre-linked Echelle spectrograph (Rao et al. 2005) attached to the 2.34-m telescope of Vainu Bappu Observatory (VBO), Kavalur. On most occasions, the spectra were obtained with a slitwidth of $60-\mu \mathrm{m}$ in front of the light feeding optical fibre of $100-\mu \mathrm{m}$ in diameter. The mean spectral resolutions with and without the slit are about 50000 and 30000 , respectively. Depending on the signal-to-noise ratio of the recorded spectra and the blending caused by the variable difference in the velocities of the components, 60-160 absorption lines of the G-type component, which have well-defined cores, were measured for its radial velocities. The rvidlines task of the IRAF $^{1}$ package was used for this purpose. Since the undersized CCD detector used did not cover the whole free spectral range, there were gaps in the recorded spectra, which varied from about $30 \AA$ to $50 \AA$ over the $4600-6700 \AA$ spectral region used for the velocity measurements. The heliocentric radial velocities of the G-type component measured by us are listed in Table 1. The probable errors in the radial velocities given in the table are smaller than $0.2 \mathrm{~km} \mathrm{~s}^{-1}$. The standard deviation of a single line measurement of radial velocity varies between $1.5 \mathrm{~km} \mathrm{~s}^{-1}$ and $2.5 \mathrm{~km} \mathrm{~s}^{-1}$ caused by the variable blending of the spectra of the G-type star with that of its companion. The radial velocity of $\alpha$ Boo measured from its spectra obtained on 18 April 2009 is $-4.9 \pm 0.1 \mathrm{~km} \mathrm{~s}^{-1}$, and the standard deviation of a single line measurement is $1.6 \mathrm{~km} \mathrm{~s}^{-1}$. The General Catalogue of Stellar Radial Velocities (Wilson 1953) gives a value of $-5.2 \mathrm{~km} \mathrm{~s}^{-1}$ for $\alpha$ Boo. Even at the extrema of the radial velocity variation, only a few well-defined absorption lines of the K-type component are available. Because of the rotational broadening and distortion by starspots, these lines are not very suitable for radial velocity measurement with the rvidlines task.

The $B V$ photometric observations of V711 Tau were obtained on a total of 202 nights during 1993-94 and 2001-09

\footnotetext{
1 IRAF (Image Reduction and Analysis Facilities) is distributed by the National Optical Astronomy Observatories, which are operated by the Association of Universities for Research in Astronomy, Inc., under cooperative agreement with the National Science Foundation.
} 
Table 1. Heliocentric velocities of the G-type component of V711 Tau.

\begin{tabular}{lrlr}
\hline \hline JD & Radial & JD & Radial \\
$2450000+$ & velocity & $2450000+$ & velocity \\
\hline 3050.1274 & -60.25 & 4821.1084 & -76.32 \\
3053.1466 & -70.20 & 4823.2145 & -3.88 \\
3054.1034 & -5.68 & 4826.2317 & -27.42 \\
3286.4015 & -57.60 & 4827.1532 & -62.63 \\
3338.1913 & 33.01 & 4831.0710 & 48.82 \\
3339.3121 & -25.84 & 4833.1146 & -28.83 \\
3340.1926 & -67.03 & 4836.1116 & -7.87 \\
3373.2103 & -6.55 & 4838.1274 & -76.35 \\
3374.1998 & -69.88 & 4844.1707 & -64.61 \\
3694.2449 & -57.39 & 4848.2175 & 48.43 \\
3703.2475 & -75.54 & 4853.1532 & -7.37 \\
3705.2197 & -5.71 & 4854.1573 & 30.37 \\
3750.0913 & 46.66 & 4864.1827 & -48.61 \\
3751.0871 & -64.81 & 4866.1020 & -45.76 \\
3752.0738 & -18.08 & 4868.1296 & 46.00 \\
3753.0744 & 38.06 & 4874.1276 & 18.28 \\
4467.2264 & -7.80 & 4881.1172 & -58.08 \\
4468.2180 & 32.45 & 4882.0776 & 47.21 \\
4811.2631 & 47.71 & 4883.1073 & -43.16 \\
4817.2468 & 32.41 & 4884.0809 & -44.38 \\
4820.1876 & 22.51 & 4885.0759 & 47.89 \\
\hline
\end{tabular}

with the 34-cm telescope of VBO. The fainter visual companion at a separation of 6 arcsec was always included in the photometric measurements. All the measurements were made with respect to the comparison star 10 Tau. Table 2 (full table available only in electronic form) lists the results of the differential photometry. Each value given in the table is an average of 3-4 independent measurements, and the typical uncertainty in the value is around $0.01 \mathrm{mag}$.

\section{Modulation of orbital period}

The radial velocities given in Table 1 are plotted in Fig. 1 after converting the Julian dates of observation to orbital phases using the following ephemeris given by Fekel (1983)

$\mathrm{JD}=2442763.952+2 \mathrm{~d} .83774 \mathrm{E}$,

where the initial epoch corresponds to the time of maximum radial velocity of the K-type primary. Different symbols are used to identify the observations obtained at different epochs. It is clear that there is a systematic shift in the velocity curve towards shorter orbital phases with time, indicating that the above assumed period is slightly longer than the true orbital period. We derived the time of maximum and the amplitude of the radial velocity variation of the G-type secondary, and the velocity of the centre of mass of the binary, individually, from the data obtained during the three observing seasons, namely, 2004-05 (JD 2453 286-374), 2005-06 (JD 2453694-753) and 2008-09 (JD 245811-885), and the results are given in Table 3. The eccentricity was assumed to be zero and the orbital period was assumed to be the same as that given by Fekel (1983). The table also gives the standard deviation of the residual to the best fit $(\sigma)$ to the data in each case. The comparatively large values of $\sigma$ for the first two data sets are obviously due to the rather small number of measurements they contain. The difference in the observed time of velocity maximum and that computed using the above ephemeris, $\mathrm{O}-\mathrm{C}$, is also given in the table. The probable error in the time of maximum radial velocity $\left(T_{\max }\right)$ is the same as that in the respective $\mathrm{O}-\mathrm{C}$ value.

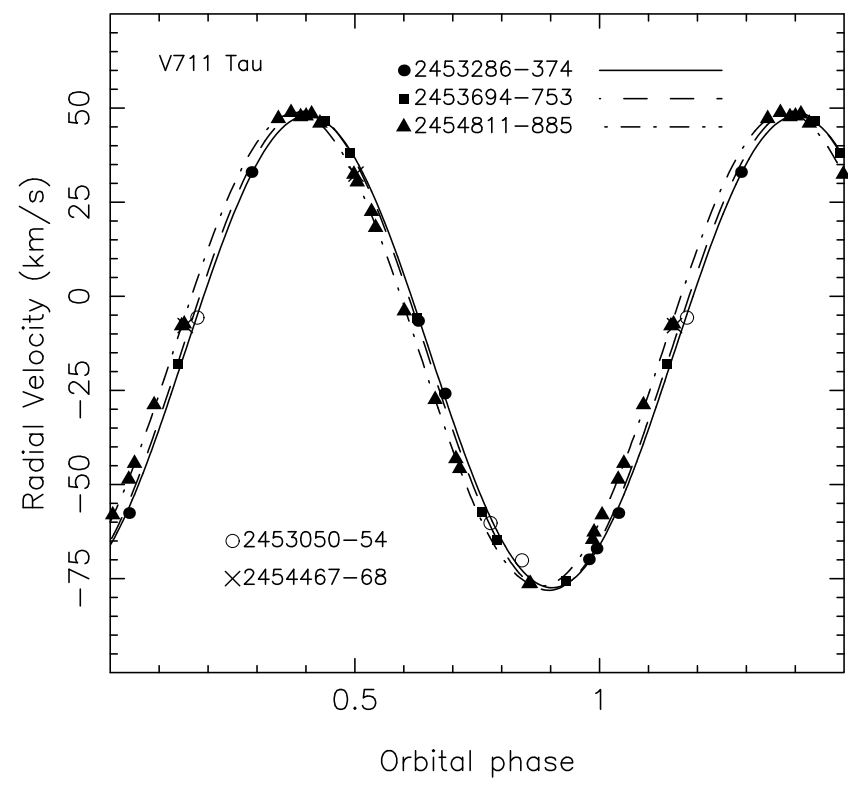

Fig. 1. Radial velocity curves of the G-type component in V711 Tau binary. The orbital phases were computed using the ephemeris JD = $2442763.952+2$ d.83774 E.

The $\mathrm{O}-\mathrm{C}$ values given in Table 3 are plotted in the top panel of Fig. 2 against the corresponding mean Julian days of observation, along with the $\mathrm{O}-\mathrm{C}$ values listed by Frasca \& Lanza (2005), which were also computed using the ephemeris given above. The plot also contains two more $\mathrm{O}-\mathrm{C}$ values: one calculated from the time of maximum, which we derived from the radial velocities reported by Gondoin (1986) from the measurements of the absorption components of Fe I lines, $6546 \AA$ and $6569 \AA$, and the other calculated from the time of conjunction given by Zhai \& Zhang (1996). The present data clearly indicate that sometime around 2002 there was a break in the steep decrease in O-C, which started around 1990. Since there is a possibility of a periodical variation in $\mathrm{O}-\mathrm{C}$, as indicated by the present data and the analysis of Frasca \& Lanza (2005), we made a non-linear least squares fit to all the available $\mathrm{O}-\mathrm{C}$ data using the somewhat more general equation of

$\mathrm{O}-\mathrm{C}=\Delta T_{\mathrm{o}}+N \Delta P_{\mathrm{o}}+\tau \cos \left[\left(2 \pi / P_{l}\right)\left(t-T_{l}\right)\right]$,

where $\Delta T_{\mathrm{o}}$ is the offset in the initial epoch used, $N$, the number of orbital cycles elapsed, $\Delta P_{\mathrm{o}}$, the correction to the orbital period, $\tau$, the amplitude of $\mathrm{O}-\mathrm{C}$ variation, and $P_{l}$ and $T_{l}$, the period and time of maximum of the $\mathrm{O}-\mathrm{C}$ modulation. The results of the least squares solution are given in Table 4.

The $\mathrm{O}-\mathrm{C}$ values were recomputed using the following revised ephemeris for the orbital radial velocity variation

$\mathrm{JD}=2442764.012+2 \mathrm{~d} .8376658 \mathrm{E}$,

where the initial epoch, again, corresponds to the maximum of the radial velocity of the K-type component, and are plotted in the bottom panel of Fig. 3 along with the computed sinusoidal curve. It is clear that a slight reduction in the orbital period used in the literature so far would result in a near sinusoidal variation in the $\mathrm{O}-\mathrm{C}$ values.

The $\Delta P / P$ derived from the observed $\mathrm{O}-\mathrm{C}$ variation, which shows an amplitude of $3.9 \times 10^{-5}$, is also plotted in the bottom panel of Fig. 3. The period, which was increasing when the regular spectroscopic observations of V711 Tau began in 1976, attained its longest value around 1983. The variation in O-C resulting from such an increase was nearly compensated for by the 
Table 2. Sample of the table of $B V$ photometry of V711 Tau.

\begin{tabular}{|c|c|c|c|c|c|c|c|c|c|c|c|}
\hline $\mathrm{JD}$ & & & $\mathrm{JD}$ & & & $\mathrm{JD}$ & & & $\mathrm{JD}$ & & \\
\hline $2400000+$ & $\Delta V$ & $(B-V)$ & $2400000+$ & $\Delta V$ & $\Delta(B-V)$ & $2400000+$ & $\Delta V$ & $\Delta(B-V)$ & $2400000+$ & $\Delta V$ & $\Delta(B-V)$ \\
\hline 49012.083 & 1.563 & 0.323 & 52329.143 & 1.496 & 0.323 & 53049.106 & 1.563 & 0.406 & 54152.084 & 1.642 & 0.336 \\
\hline 49014.156 & 1.599 & 0.370 & 52330.131 & 1.487 & 0.384 & 53055.098 & 1.613 & 0.367 & 54153.106 & 1.602 & 0.336 \\
\hline
\end{tabular}

Table 3. Orbital elements of the G-type component of V711 Tau.

\begin{tabular}{cccc}
\hline \hline & JD 2 453000+ & JD 2 453 000+ & JD 2 454 000+ \\
Parameter & $286-374$ & $694-753$ & $811-885$ \\
\hline$T_{\max }$ & 341.353 & 730.104 & 848.128 \\
$K\left(\mathrm{~km} \mathrm{~s}^{-1}\right)$ & $62.7 \pm 0.6$ & $63.6 \pm 0.4$ & $62.9 \pm 0.1$ \\
$\gamma\left(\mathrm{km} \mathrm{s}^{-1}\right)$ & $-14.7 \pm 0.4$ & $-14.5 \pm 0.3$ & $-14.2 \pm 0.1$ \\
$\sigma\left(\mathrm{km} \mathrm{s}^{-1}\right)$ & 1.4 & 1.0 & 0.7 \\
$\mathrm{O}-\mathrm{C}$ & $-0.274 \pm 0.004$ & $-0.293 \pm 0.003$ & $-0.340 \pm 0.001$ \\
\hline
\end{tabular}

Table 4. Results of the least square solution of the $\mathrm{O}-\mathrm{C}$ data of V711 Tau.

\begin{tabular}{cc}
\hline \hline Parameter & Value \\
\hline$T^{\max }$ (orbit) & JD 2 442 764.012 \pm 0.012 \\
$P$ (orbit) & $2.8376658 \pm 0.0000039 \mathrm{~d}$ \\
$T^{\max }$ (long-term) & JD $2448195 \pm 134$ \\
$P$ (long-term) & $36.3 \pm 1.9 \mathrm{yr}$ \\
$($ O-C) amplitude & $0.083 \pm 0.005 \mathrm{~d}$ \\
\hline
\end{tabular}

slightly longer assumed orbital period, and thereby, produced the near-constancy in O-C from about 1976 to 1990, seen in the top panel of Fig. 2. When the period started decreasing, the change in $\mathrm{O}-\mathrm{C}$ produced was in the same direction as that produced by the assumed longer orbital period to compute it, and hence, resulted in its steep decrease, observed subsequently.

The velocities of the centre of mass of the V711 Tau binary system $(\gamma)$ determined over 1976-2009 by various authors are plotted in the bottom panel of Fig. 2. There is obviously no appreciable long-term modulation in $\gamma$; it is constant to well within $\pm 1 \mathrm{~km} \mathrm{~s}^{-1}$. The couple of velocities that deviate significantly from the mean of $-14.5 \pm 0.1 \mathrm{~km} \mathrm{~s}^{-1}$ are those measured by Frasca \& Lanza (2005) using the IUE data, and according to them the IUE velocities may be affected by systematic errors. The expected variation in $\gamma$, if the sinusoidal variation seen in $\mathrm{O}-\mathrm{C}$ is as a result of light-time effect caused by the orbital motion of V711 Tau about an unseen companion, is also plotted in the bottom panel of Fig. 2. As pointed out by Frasca \& Lanza (2005) the O-C variation shown by V711 Tau is definitely not caused by any light-time effect. The amplitude of $\pm 0.083 \mathrm{~d}$ in O-C over 36.3-yr would imply an amplitude of $\pm 11.8 \mathrm{~km} \mathrm{~s}^{-1}$ for the radial velocity variation of the centre of mass.

\section{Spot activity}

\subsection{Activity cycle}

The $\Delta V$ values given in Table 2 are plotted in the top panel of Fig. 3 along with those listed by Lanza et al. (2006) and Berdyugina \& Henry (2007), after converting them to $V$ using $V_{10 \text { Tau }}=4.28$ mag, against the corresponding Julian days of observation. Following Lanza et al. (2006), from the measurements of V711 Tau listed in Table 2 and those listed by Berdyugina \& Henry (2007), we removed the contribution of the visual companion before plotting them in the figure, using $V=8.83 \mathrm{mag}$ for the star.

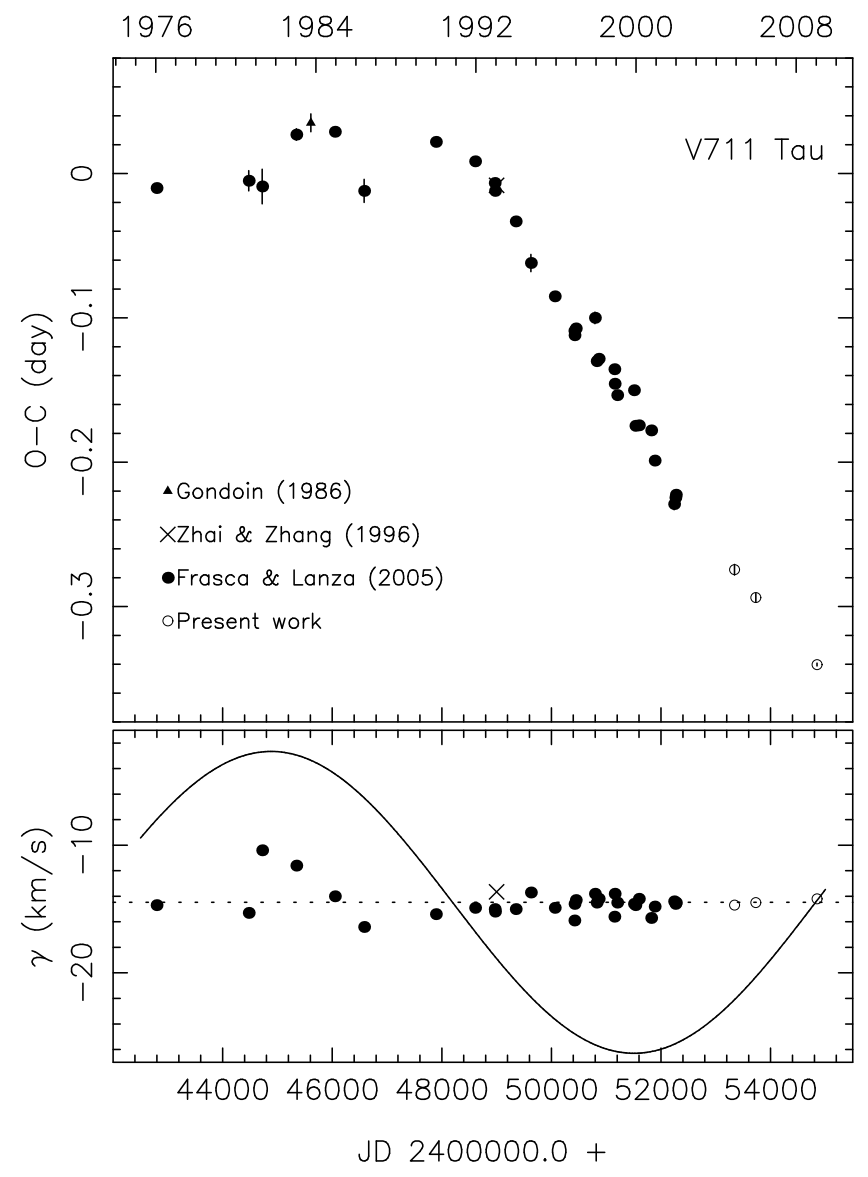

Fig. 2. Top panel: the $\mathrm{O}-\mathrm{C}$ variation of V711 Tau. The times of maximum radial velocity were computed using the ephemeris JD = $2442763.952+2$ d 83774 E. Bottom panel: the observed centre of mass velocity. The expected variation, if the $\mathrm{O}-\mathrm{C}$ resulted from light-time effect due to the orbital motion about an unseen companion, is plotted as the continuous curve. The $\mathrm{O}-\mathrm{C}$ values used for this are those computed using the revised ephemeris JD $=2442764.012+2.8376658 \mathrm{E}$.

The photometry obtained during the recent observing seasons shows that the mean brightness of V711 Tau is in a recovery phase from a deep minimum, which is indicative of a continuous reduction in its spot activity observed during the previous seasons. Berdyugina \& Henry (2007), who analysed the extensive photometric data over the years 1975-2006 with an inversion technique, concluded that there are two active longitudes, with long-lived starspots, that migrate in the orbital reference frame with variable rates as a result of the continuous changes in their mean latitudes on the differentially rotating active star. They also concluded that these active regions regularly approach each other and merge into a large single region, and at these epochs produce large amplitudes in the light variation. Their model predicted a merger in 2007, but the photometry obtained by us during 2007-08 shows no such increase in the amplitude of light variation around that time. 


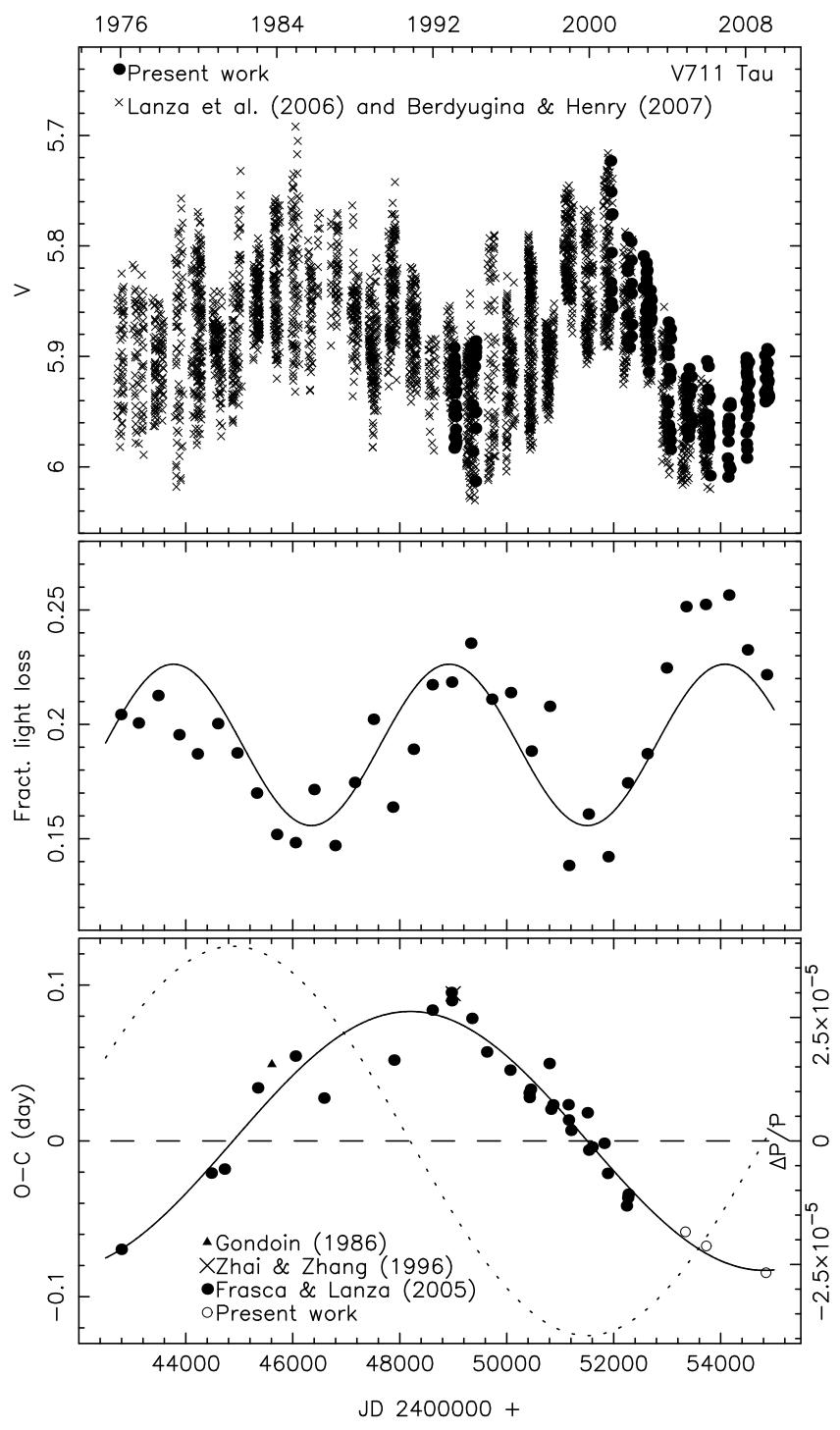

Fig. 3. Top panel: plot of the $V$ mag of V711 Tau against the corresponding Julian day of observation. The contribution by the faint visual companion was removed from the observed brightness. Middle panel: plot of fractional loss of light over a photometric cycle against the corresponding mean epoch of observations. The continuous line represents a least squares sinusoidal fit to the data; the time of maximum and period are JD 2448925 and 5155-d, respectively. Bottom panel: plot of the $\mathrm{O}-\mathrm{C}$ values computed using the revised ephemeris JD = $2442764.012+2.8376658 \mathrm{E}$. The continuous line represents the computed $\mathrm{O}-\mathrm{C}$ using the amplitude, period, and time of maximum of the long-term modulation given in Table 4 . The dotted line shows the variation in $\triangle P / P$ derived from the revised $\mathrm{O}-\mathrm{C}$ variation.

The $V$ magnitudes plotted in Fig. 3 clearly show that the mean brightness of V711 Tau has a long-term modulation, indicating a corresponding long-term modulation in the spot activity. The fractional loss of light integrated over a photometric cycle is a better indicator of the spot activity than the mean light level because the process of integration gives weightage to the usually asymmetric light curves. The main parameter involved in the calculation of the loss of light is the reference brightness level. The choice of the reference is not very crucial in analysing the general trend in the long-term variation in spot activity since a change in the reference level would merely shift vertically the fractional light-loss computed at all epochs by the same amount. If the reference level chosen corresponds to the actual brightness of the quiescent, unspotted photosphere, the fractional loss of light calculated would correspond to the actual loss of light over the photometric cycle. In single-lined binaries, where the light contribution by the companion to the total light is negligible, the light-loss would correspond to the fractional area covered by spots on the visible surface, if they are perfectly black and the limb-darkening effects are not appreciable. The actual fractional spotted area would be significantly larger than the fractional light-loss if the temperatures of the spots are appreciable. Using synthetic light curves, Mekkaden \& Raveendran (1998) demonstrated that the effect of limb-darkening on the observed brightness would depend on the exact distribution of spots on the stellar surface, and that the effects would be negligible for spot distributions with appreciable longitudinal extents over the visible hemisphere.

The $V=5.65$ mag of V711 Tau observed in 1985 is the brightest so far observed. Berdyugina \& Henry (2007) assumed the unspotted magnitude of the star to be 0.05 mag brighter than this, while Lanza et al. (2006) assumed this to correspond to the unspotted brightness. Following Lanza et al. (2006), we used $V=5.65$ mag to be the unspotted brightness of V711 Tau, which after the removal of the contribution from the faint visual companion became 5.692 mag. We derived the fractional light-loss during each observing season from the corresponding light curve and the results are plotted in the middle panel of Fig. 3; all the available observations during a particular season were used in the computation of the light-loss over a photometric cycle during that season.

From the $V$ band photometry of V711 Tau from 1975 to 2001, using inversion techniques, Lanza et al. (2006) derived the fractional spot area on the surface of the K-type component. To overcome the problems of non-uniqueness and instabilities inherent in the inversion of wide-band photometric data, they introduced regularisation functions in their inversion method, and found that the Maximum Entropy function gives a systematically smaller spot area than the Tikhonov regularisation function; the fractional spot areas during 1975-2001 given by the former function lie in the range $0.06-0.12$, while that given by the latter lie in the range $0.13-0.27$. The fractional loss of light during the corresponding period derived by us shows a variation very similar to that shown by the spot area derived by Lanza et al. (2006). From the middle panel of Fig. 3 we find that the fractional light-losses during 1975-2001 derived by us lie in the range $0.14-0.24$. In their spot modelling, Lanza et al. (2006) assumed the difference in the magnitudes of the components of the binary system at the unspotted epoch to be 1.4. If we take into account the contribution by the secondary to the total light, the fractional loss of light by the active K-type primary would lie approximately in the range $0.18-0.30$, and if we account for the spots not being perfectly black, the range of spot area would be significantly greater than this, indicating that the Tikhonov regularisation function used by Lanza et al. (2006) also predicts significantly smaller spot areas than actually existing on the active star.

The variation in fractional light-loss, and hence, in spot area over the surface of the star, seems to be more cyclic than strictly periodic in nature. The available data base-line is not long enough relative to the timescales involved in the variation to make any conclusive remarks. To date, we have observed only two minima and three maxima of the spot activity cycle. The fractional light-losses observed at the two minima are not very much different, while those at the maxima suggest the possibility of a long-term modulation. It may be noted that even at the minimum spot activity a substantial fraction of the stellar surface is 


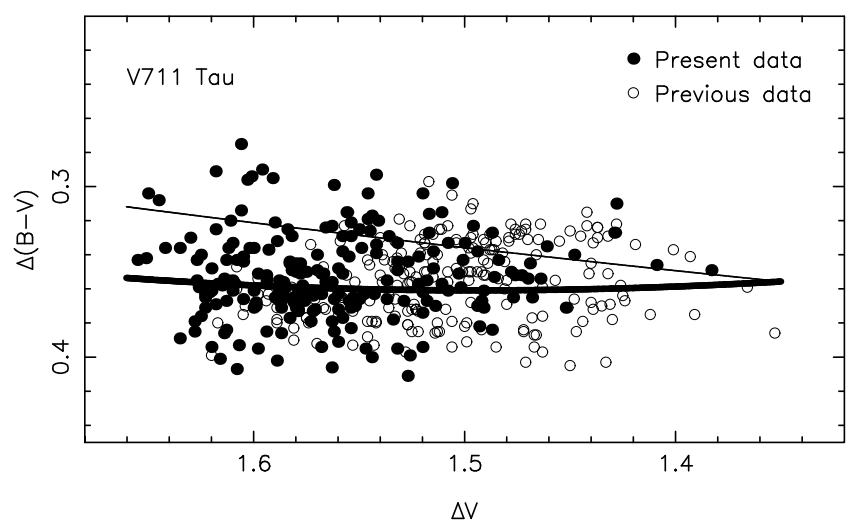

Fig. 4. Plot of $\Delta(B-V)$ value of V711 Tau with respect to 10 Tau against the corresponding $\Delta V$ obtained at VBO over 1977-2009. The previous data are from Parthasarathy et al. (1981), Mekkaden et al. (1982), and Mohin \& Raveendran (1993). The thick line represents the expected variation in the composite $(B-V)$ colour of V711 Tau if the $(B-V)$ of the active star varied from 1.08 to $1.20 \mathrm{mag}$ when $\Delta V$ varied from 1.35 to $1.65 \mathrm{mag}$, and the thin line represents the case if $(B-V)$ of the active star remained constant at $1.08 \mathrm{mag}$. The $(B-V)$ of the G-type component was assumed to be $0.75 \mathrm{mag}$. The effect of the presence of the faint visual companion was taken into account while computing the $(B-$ $V)$ variation.

covered by spots. An average period of $14.1 \pm 0.3$ yr satisfies the observed variation in light-loss. This period is consistent with the 15 -yr period of variation in the mean brightness reported by Berdyugina \& Henry (2007) and the $19.5 \pm 2.0 \mathrm{yr}$ period of the variation in the spot area derived by Lanza et al. (2006) from a smaller base-line data set.

\section{2. $(B-V)$ colour variation}

In Fig. 4, we plot the $\Delta(B-V)$ values of V711 Tau obtained at VBO with the same photomultiplier-filter set up during 1977-2009 against the corresponding $\Delta V$ values. The VBO data cover the full range in $V$ magnitudes of V711 Tau observed so far. There is no clear indication from the data that the star becomes bluer when it is fainter, as reported by Ulvas \& Henry (2005). The mean $\Delta(B-V)$ is apparently independent of $\Delta V$ in the range covered by the observations. A least squares fit to the data plotted in Fig. 4, however, shows that there is a slight tendency for the star to become redder as it becomes fainter. The $\Delta(B-V)$ shows a scatter of about $0.1 \mathrm{mag}$, which is significantly larger than the observational errors, as in the case of the other active RS CVn binaries, UX Ari, II Peg, and DM UMa (Rosario et al. 2007).

The $(B-V)$ data plotted in Fig. 4 are not consistent with the general understanding that the light modulations in RS CVn binaries are caused by starspots that are cooler than the ambient photosphere, in which case we would have observed a redder colour for V711 Tau at fainter visual magnitudes. From an analysis of an extensive multicolour photometry of the single-lined, active RS CVn binary DM UMa, Rosario et al. (2009) found that there are excess fluxes in $U$ and $B$ bands, which increase as the star becomes fainter, but they compensate only partially for the greater reduction in flux in those bands caused by spot activity.

With $(B-V)=0.58$ for 10 Tau (Hoffleit \& Jaschek 1982), the mean $\Delta(B-V)=0.354$ obtained from our observations yields $(B-V)=0.934$ for V711 Tau, which is in close agreement with $(B-V)=0.92$ given by both Eggen (1978) and Dean (1979) for the star. We computed the $(B-V)$ colours of the active star that

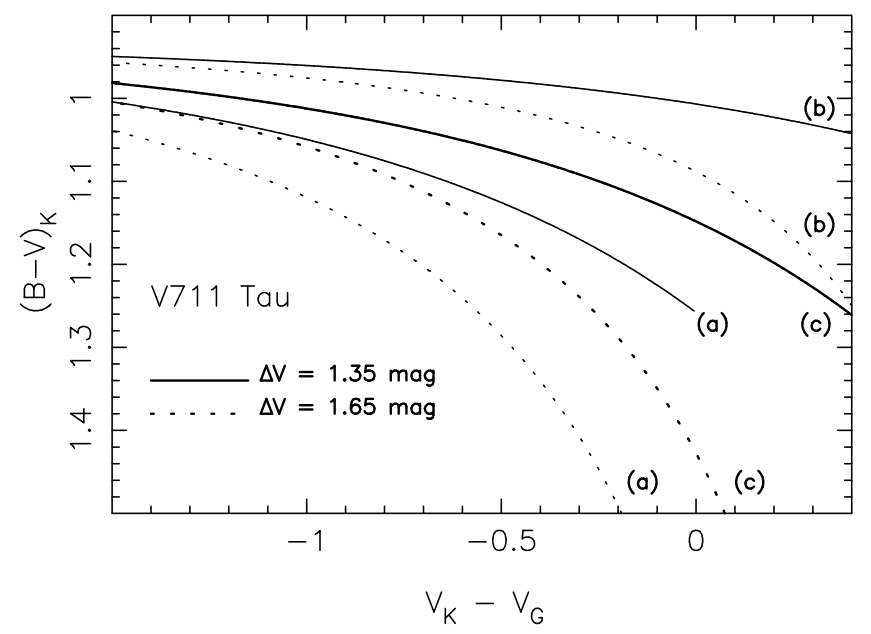

Fig. 5. Plots of the derived $(B-V)$ colours of the active star at the brightest and the faintest $V$ magnitudes of V711 Tau observed against the corresponding assumed difference in the $V$ magnitudes of the unspottedactive star and the G-type component: $(a),(b)$, and $(c)$ represent the cases where the $(B-V)$ of the G-type star was assumed to be 0.68 , 0.86 , and 0.75 , respectively.

would produce the above mean composite colour of V711 Tau as it varied from $\Delta V=1.35$ to $\Delta V=1.65$ for a range of assumed differences in the $V$ magnitudes of the unspotted active star and the G-type component, $V_{K}-V_{G}$. The computations were made for different $(B-V)$ colours for the G-type component: (a) 0.68 , (b) 0.86 , and (c) 0.75 . The first two colours correspond to those of dwarfs and giants of spectral type G5 (Drilling \& Landolt 2000), and the results are plotted in Fig. 5. We removed the contribution from the faint visual companion to the observed $V$ and $(B-V)$ values using $V=8.83$ and $(B-V)=0.99$ for it (Eggen 1966). From the figure, it is clear that, to produce the observed composite $(B-V)$ colour, even beyond a magnitude difference of -1.5 , the active star has to be redder when it is fainter for all possible $(B-V)$ colours of the G-type component; this is surely consistent with the conventional starspot hypothesis. The excess reduction in the $B$ band flux over the $V$ band flux of the active component due to cool-spot activity is nearly nullified by the fractional increase in the contribution to the total $B$ band flux by the hotter companion, giving rise to a nearly constant composite $(B-V)$ colour for V711 Tau.

On the basis of the nearly equal strengths of the absorption lines of the components in the V711 Tau binary system, Bopp $\&$ Fekel (1976) pointed out that their luminosities are, probably, nearly equal in the blue region. Popper (1978) and Fekel (1983) suggested that the difference in the $B$ magnitudes of the components, $B_{K}-B_{G}$, most likely, lies in the range -0.2 to 0.0 mag. Fekel's observations were performed during 1975-81, and from the top panel of Fig. 3, we find that V711 Tau was, on the average, about 0.2 mag below its maximum brightness ever observed. Taking the difference in colours of the components, $(B-V)_{K}-(B-V)_{G}$, to be $0.3 \mathrm{mag}$, using the colours appropriate to their spectral types, the above range in difference in $B$ magnitudes would imply a range of -0.6 to -0.8 for the difference in the $V$ magnitudes of the components, $V_{K}-V_{G}$, at the epoch of maximum brightness of V711 Tau.

Because of the large rotational broadening of the absorption lines of the active star and similar spectral types of the components of the binary system, generally, only a few unblended lines are available for equivalent width (EW) measurements even 
Table 5. Equivalent widths of absorption lines.

\begin{tabular}{ccc}
\hline \hline Object & $6430.84(\mathrm{Fe} \mathrm{I})$ & $6439.07(\mathrm{Ca} \mathrm{I})$ \\
& $\AA$ & $\AA$ \\
\hline HD 138716 (K1 IV) & 0.172 & 0.191 \\
HD 152311 (G5 IV) & 0.126 & 0.166 \\
HD 59468 (G5 V) & 0.123 & 0.166 \\
G-type component & $0.064 \pm 0.002$ & $0.078 \pm 0.001$ \\
$\alpha$ Boo (K1 III) & 0.213 & 0.217 \\
\hline
\end{tabular}

at the extrema of radial veocity variations, when the absorption lines of the components are well separated. In our case, the gaps in the recorded spectra reduce the number of available unblended lines. We measured the EWs of $6430.84 \AA$ (Fe I) and $6439.07 \AA$ (Ca I) lines of the G-type component of the V711 Tau binary system from 13 of the spectra obtained by us and the averages of these are given in Table 5. The EWs were measured by fitting Gaussian profiles to the absorption lines. The table also contains the EWs of the same spectral lines of HD 138716 (K1 IV), HD 152311 (G5 IV), HD 59468 (G5 V), and $\alpha$ Boo (K1 III), again, derived by fitting Gaussian profiles to their absorption lines. The spectra of the first three stars were downloaded from Bagnulo et al. (2003) and that of the last was obtained at VBO. The EWs of the $\mathrm{Ca}$ I line of $\alpha$ Boo given in the table agree well with those given by Hatzes et al. (1996) for stars of comparable spectral types. The IRAF task splot was used for the measurement of the EWs.

Assuming the actual EWs of the absorptions lines of the G-type component of the V711 Tau binary system to be the same as the averages of the G5 stars given in Table 5, from the observed EWs of $6430.84 \AA$ and $6439.07 \AA$ absorption lines, we find the difference in magnitudes (mag of K-type star minus mag of G-type star) at these wavelengths to be $+0.07 \pm 0.06$ and $-0.14 \pm 0.03$. The difference in the effective temperatures of the components, $4800 \mathrm{~K}$ and $5400 \mathrm{~K}$ (Ulvas \& Henry 2005), would make the active K-type star relatively fainter by $\sim 0.1$ mag than the G-type star in $V$ band. Therefore, we find the average $V_{K}-V_{G}$ to be $0.06 \pm 0.05 \mathrm{mag}$ when the $V$ magnitude of V711 Tau (after the removal of the contribution from the faint visual companion) varied between 5.99 and 5.90 , and $V_{K}-V_{G}$ to be $-0.4 \pm$ $0.1 \mathrm{mag}$ at the unspotted $V$ magnitude of 5.692; the latter value is comparable to the rough estimate presented above, based on the suggestions of Popper (1978) and Fekel (1983) from the intensities of the absorption lines of the component stars in the photographic region. The $V$ luminosity ratio of the components of V711 Tau suggested by Donati et al. (1992) would have made the EWs of the above lines of the G-type component at least $30 \%$ smaller than that observed by us.

In Fig. 4, along with the observations, we plot the expected variations in the composite colour of V711 Tau assuming that: (i) the $(B-V)$ of the active star varied from about 1.08 to 1.20 and (ii) the $(B-V)$ remained constant at 1.08 , the colour at the light maximum, when its $\Delta V$ varied by $0.30 \mathrm{mag}$; these $(B-V)$ colours correspond to the case $V_{K}-V_{G} \sim-0.4 \mathrm{mag}$ and $(B-V)=0.75$ for the hotter component (Fig. 5). It is clear from Fig. 4 that the active star has to be redder when fainter if the composite colour has to remain nearly constant and that even a constant $(B-V)$ for the active star during its brightness variation would make the composite colour appreciably bluer when it becomes fainter.

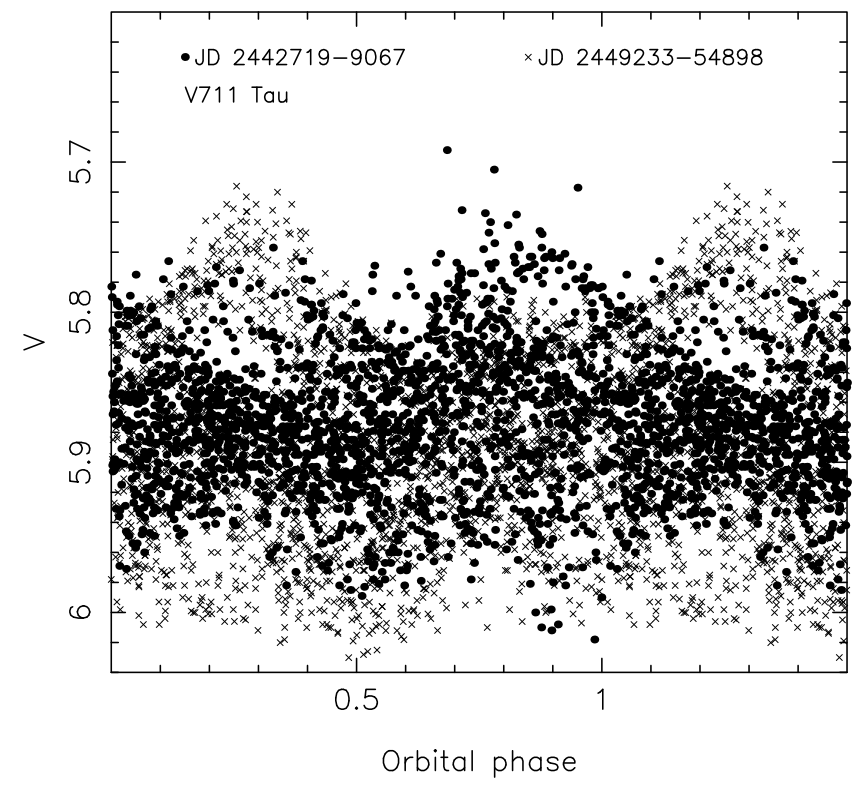

Fig. 6. Plot of $V$ mag of V711 Tau against the corresponding orbital phase computed using the ephemeris JD $2442766.140+2.8376658 \mathrm{E}$.

\subsection{Orbital modulation of spot activity}

Rosario et al. (2008) reported that the differential $V$ mag of UX Ari available from 1972 to 2008, when folded over the orbital period, has a spread of about 0.48 mag around $0.50 \mathrm{p}$ against a spread of only $0.18 \mathrm{mag}$ around $0.0 \mathrm{p}$; we find that the most recent data are also consistent with this. The $0.0 \mathrm{p}$ corresponds to the conjunction with the active star in front. Assuming that the spread in the $V$ mag is an indicator of spot activity, in the sense that, spots form and disintegrate more often, the above authors pointed out that UX Ari apparently has a longitudinal asymmetry in spot activity that is fixed in the orbital frame of reference, with the hemisphere facing the hotter companion being more active than that facing away from it.

In Fig. 6, we plot all the $V$ band observations of V711 Tau shown in the top panel of Fig. 3 after folding them over its orbital period using the ephemeris

$\mathrm{JD}=2442766.140+2 \mathrm{~d} .8376658 \mathrm{E}$,

where the initial epoch corresponds to the conjunction with the active star in front and the period is the revised orbital period given in Table 4. The initial epoch is calculated from the revised epoch of maximum radial velocity, which is also given in the same table. In contrast to that of UX Ari, the orbital period of V711 Tau varies significantly. However, the bottom panel of Fig. 3 and Table 4 show that the maximum deviation in the time of conjunction as a result of the variation in its orbital period is only $\pm 0.083 \mathrm{~d}$, which would translate into an uncertainty of only \pm 0.029 in phase.

The $V$ band data were divided into two subsets, with those obtained before the maximum of the spot activity cycle that occurred around 1994 being placed into one set and the rest into the other, and are plotted with separate symbols in Fig. 6. From the figure we find that there is a suggestion that the spot activity has a component that is fixed in the orbital frame of reference; the upper envelope of the $V$ magnitudes shows a maximum around each of the quadratures, $0.25 \mathrm{p}$ and $0.75 \mathrm{p}$. The brighter $V$ magnitudes obtained during JD 2442 719-9067 are distributed mostly around 0.75 , while those obtained during JD 244923-54898 are distributed around $0.25 \mathrm{p}$. We would expect the $V$ magnitudes 
to be fairly uniformly distributed across all orbital phases when data spanning about 4300 photometric cycles are folded over the rotational period of the active star if the spots form and disintegrate randomly across all its longitudes; the differential rotation of the active star, if present, would make the distribution of the $V$ magnitudes uniform across the orbital phases.

\subsection{Ho emission}

Of the 24 spectra obtained during the 2008-09 observing season, only 21 spectra cover the $\mathrm{H} \alpha$ region. Using the IRAF task dopcor, the spectra were shifted to the rest frame of the active star before continuum fitting. The radial velocities of the active star were computed using $\gamma=-14.48 \mathrm{~km} \mathrm{~s}^{-1}, K_{K}=$ $49.04 \mathrm{~km} \mathrm{~s}^{-1}$, and $T_{\max }=$ JD 2454846.709 . The values of $\gamma$ and $K_{K}$ are the averages of all the observed values, and $T_{\max }$ is that derived using the time of maximum of radial velocity of the G-type component observed in 2008-09 (Table 4). We measured the equivalent width of $\mathrm{H} \alpha$ emission above continuum (EW), the centroid of wavelength of emission $\left(\lambda_{0}\right)$, and the base-line width (BLW) of the emission profile from these spectra using the IRAF task splot, and the results are presented in Fig. 7 along with the contemporaneous $\Delta V$ magnitudes given in Table 2. The wavelength centroid corresponds to the intensityweighted wavelength of the emission feature above the continuum, and its deviation $\left(\Delta \lambda_{0}\right)$ from the rest wavelength $6562.82 \AA$ is plotted in the figure. The orbital phases were computed from the Julian days of observation using the revised ephemeris.

The EWs of $\mathrm{H} \alpha$ emission plotted in Fig. 7 do not show any obvious orbital modulation. But, there is an indication that during the phases of minimum light the EWs show a larger range of variation, which causes the upper envelope of the observed EWs to appear to be anti-correlated with the light variation. The largest EW of $3.55 \AA$ observed at JD 2454821.1085 (0.199p) is, most likely, due to an intense flare activity. The BLW of emission on that occasion was also high, $\sim 11.2 \AA$. If we do not take the flare event into account, there is an indication from the figure that the BLWs of $\mathrm{H} \alpha$ emission show a larger fluctuation during the phases of minimum light $(0.5-1.1 \mathrm{p})$ than during the phases of maximum light $(0.1-0.5 \mathrm{p})$. The values of $\Delta \lambda_{0}$ plotted in Fig. 7 do not show any modulation with the orbital phase. If the secondary contributed to the $\mathrm{H} \alpha$ emission, as suggested by Fraquelli (1982), we would have seen a clear orbital modulation in the values of $\Delta \lambda_{0}$. The entire $\mathrm{H} \alpha$ emission can be attributed to the K-type active star, as previously proposed by Gondoin (1986) and Montes et al. (1997).

\section{Discussion}

The modulation in orbital periods observed in RS CVn stars (Hall 1990), in general, requires a cyclic mechanism internal to the binary system. The mechanism proposed by Applegate (1992), where the orbital motion is gravitationally coupled to variations in the shape of the magnetically active star due to variations in its internal angular velocity as it goes through its activity cycle, was later found to be inadequate on both observational and theoretical grounds (Lanza \& Rodono 2004; Lanza 2005). The most direct prediction of the Applegate mechanism is that the modulations in the orbital period and spot activity should have the same timescale. The analysis of the radial velocity data of V711 Tau presented in Sect. 3 indicates that the $\Delta P / P$ has a near-sinusoidal modulation with a period around $36.3 \pm 1.9 \mathrm{yr}$, while the spot activity varies with an average

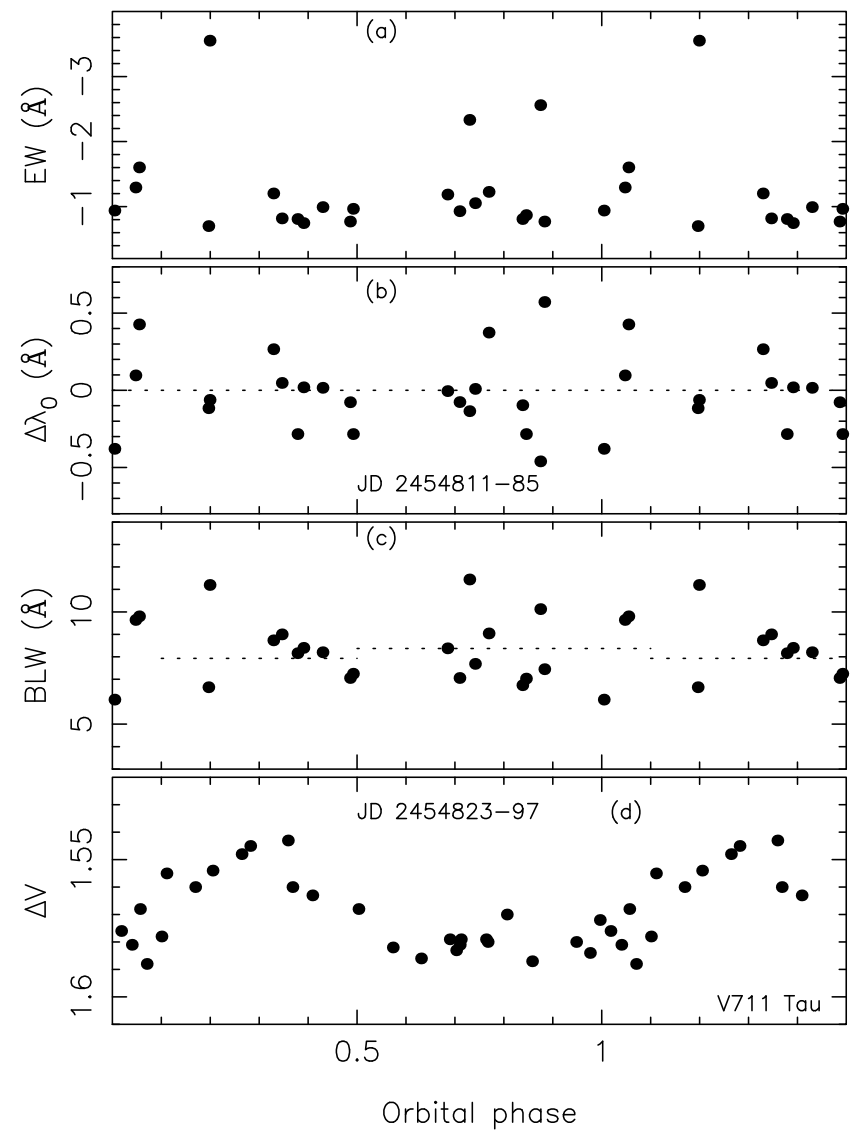

Fig. 7. Plots of $\mathrm{H} \alpha$ emission equivalent width a), shift in the centroid of wavelength of $\mathrm{H} \alpha$ emission from the rest frame wavelength b), base-line width of $\mathrm{H} \alpha$ emission c), and differential $V$ magnitude d) against the corresponding orbital phase. The dotted lines in panel c) show the averages of the base-line widths observed over the respective phase intervals. The orbital phases were computed using the ephemeris JD $2442766.140+2.8376658 \mathrm{E}$.

period around $14.1 \pm 0.3 \mathrm{yr}$, which is clearly inconsistent with the mechanism.

Lanza et al. (1998) and Lanza \& Rodono (2004) suggested improvements to the Applegate's mechanism, where they proposed that the anisotropic magnetic tension associated with the toroidal magnetic field also plays a role in distorting the shape of the star. According to their model, the magnetic energy, which is a measure of the total spot area, goes through two maxima during a cycle of orbital period variation. The modulations in fractional light-loss and $\triangle P / P$ plotted in the middle and bottom panels of Fig. 3 are consistent with their prediction. The ratio of the period of spot activity cycle to that of orbital period modulation that we derive from the present analysis is $2.54 \pm 0.14$.

UX Ari, another bright member of the group of active RS CVn binaries, is very similar to V711 Tau, apart from in terms of its orbital period, which is about 2.25 longer. The primaries and secondaries of both the binaries have similar spectral types, K0-K1IV and G5V, respectively, and masses (Fekel 1983; Carlos \& Popper 1971; Duemmler \& Aarum 2001). We note that the orbital period of UX Ari is fairly constant (Duemmler \& Aarum 2001) and does not show any modulation that may be related to its spot activity. The comparatively larger orbit (1.7 times larger) probably weakens the varying gravitational coupling between the component stars because of the changing quadrupole moment of the active star, thereby, producing changes in the orbital period that are difficult to detect. 
Rosario et al. (2008) pointed out that the apparent longitudinal asymmetry in spot activity that is fixed in the orbital reference frame observed in UX Ari would mean that differential rotation in the active component is either absent, or negligibly small, and that the presence of the companion significantly modulates the surface distribution of spots on the active star. The $V$ magnitudes plotted in Fig. 6 indicate that the situation is, probably, also similar in the case of the active star in V711 Tau.

Henry et al. (1995) reported that the high latitude regions in the active star of the V711 Tau binary system rotate more rapidly than the low latitude regions. They used the contemporary Doppler images to identify the latitudes of the spots involved in producing the light modulation and the orbital migration of the phase of light minimum $\left(\phi_{\min }\right)$ to determine the rotational period. Based on a spot modelling of the photometric data, Alekseev (2005) concluded that spots are confined to only the $30-40^{\circ}$ latitude region, and the active star has a solartype differential rotation; in this case also, the apparent migration of $\phi_{\min }$ in the orbital phase was used to determine the rotational period. It may be noted that $\phi_{\min }$ represents the centroid of longitudinal distribution of spots, and any change in the surface distribution will be reflected in its value, hence it is very difficult to derive the rotational period with any great certainty.

From an extensive set of Doppler images, Vogt et al. (1999) found the spots in V711 Tau to be tightly locked to the orbital frame and to show very little evidence of shear due to the differential rotation of the active star. From a few selected longlived features seen in the Doppler images of V711 Tau, they derived a slow differential rotation in the active star, in the sense that, the intermediate and low latitudes rotate slower than poles and high latitudes. From Zeeman Doppler imaging, Petit et al. (2004), however, found evidence of a comparatively faster differential rotation in the opposite sense, with the equator rotating faster than the pole as in the case of the Sun, but with the ratio of the difference in rotation rates at the equator and pole to the rotation rate at the equator being more than 40 times smaller than that of the Sun. Petit et al. (2004) attributed the very low rotational shear of the surface features detected by them to the drastic reduction in the differential rotation of the active star by the tidal forces due to its companion, as shown by the theoretical works of Scharlemann (1982). Apart from weakening the differential rotation, it is likely that the companion also significantly affects the surface distribution of spots on the active star.

Rosario et al. (2007) pointed that all the chromospherically active binaries that are known to show or suspected to show bluer colours at fainter visual magnitudes, UX Ari, V711 Tau, RS CVn (Ulvas \& Henry 2005), and MM Her (Tas et al. 1999), are double-lined spectroscopic binaries. Since there is no apparent reason for the spots on the active components of double-lined binaries to differ from those on the corresponding components in single-lined systems, we conclude that the near-constancy of the composite $(B-V)$ colour of V711 Tau as it becomes fainter is caused by the higher fractional contribution by the hotter component to the total light of the system in the blue spectral region. There may be faculae associated with the starspots as in the case of sunspots, but, they are definitely not responsible for the peculiar variation in $(B-V)$ shown by V711 Tau, as suggested by Ulvas \& Henry (2005).

While modelling the observed colour variation, Ulvas \& Henry (2005) derived the $V$ magnitudes of the unspotted Kand G-type components using the Barnes-Evans relation (Barnes \& Evans 1976), which relates the effective temperature to the unreddened $V$ magnitude and angular size of a star. Using the parameters given by them and the bolometric corrections, -0.31 and -0.07 , respectively, for the $\mathrm{K}$ - and G-type components, we find that $V_{K}-V_{G}=-1.6 \mathrm{mag}$, which is about 0.2 mag brighter than that Lanza et al. (2006) used in their light curve modelling, and $B_{K}-B_{G} \sim-1.3 \mathrm{mag}$. These values are definitely about $1 \mathrm{mag}$ brighter than the value indicated by the spectra of the component stars. In the present analysis of the strengths of the absorption lines of the secondary in V711 Tau, we have assumed its spectral type to be G5 V-IV, as done by other authors. The observed equivalent widths would imply a fainter $V$ magnitude for it, if its spectral type is later than this. In this situation the Barnes-Evans relation would also give a fainter $V$ magnitude for the secondary. V711 Tau was found to be a double-lined binary using photographic plates (Bopp \& Fekel 1976); there would have been no trace of the spectrum of the secondary had it been about 1 mag fainter than the primary (Heintz 1978). Rosario et al. (2007) reported that the discrepancy in the differences in the magnitudes of the components of UX Ari derived using Barnes-Evans relation and those suggested from spectroscopic observations is more than 2 mag. These discrepancies in the differences in magnitudes of the components derived indicate that the ratio of the radii of the components in V711 Tau and UX Ari are overestimated. For V711 Tau, the ratio of radii given by Garcia-Alvarez et al. (2003) and Donati (1999) are 3.0 and 3.36, respectively. A difference of $\sim 0.5$ in the $V$ magnitudes of the components at the maximum brightness of the active star would imply a value around 1.8 for the ratio of radii of the components. The radii of the components of V711 Tau and UX Ari were derived from their observed $v \sin i$ values (Garcia-Alvarez et al. 2003; Duemmler \& Aarum 2001) assuming that the components are in synchronous rotation with the orbital motion and the inclinations of their rotational axes are the same. The photometric modulation clearly shows that the active primary rotates in near-synchronisation with the orbital motion; but, there is no direct indication that the secondary also does similarly. Stawikowski \& Glebocki (1994) reported that the equatorial and orbital planes of active stars in synchronous long-period RS CVn stars are nearly aligned, while in asynchronous long-period RS CVn systems, they are, generally, not. Therefore, the low values of $v \sin i$ of the secondary components in these binary systems suggest that they are either rotating asynchronously, or their equatorial and orbital planes are not coplanar, or a combination of both.

An accurate determination of the EWs of the absorption lines of the active star of V711 Tau directly from the spectra is difficult because of the practical difficulty in specifying the continuum points. However, even rough measurements show that the EWs of $6430.84 \AA$ and $6439.07 \AA$ lines are very close to or even higher than those of the K1 IV star given in Table 5. The strong continuum from the secondary should have made the EWs at least 30-40\% smaller than those of HD 138716. When the spectroscopic observations were made V711 Tau was on an average 0.25 mag below its maximum brightness observed, indicating that a substantial fraction of the visible surface of the active star was covered by spots. The EWs of these lines increase at cooler temperatures, hence the cool spotted regions contribute more to the net observed EWs of these lines than the quiescent, unspotted photosphere. For an accurate estimation of the brightness ratio of the components of the binary system from the EWs of the absorption lines of the active star, the exact surface temperature distribution of the star is required.

Zhai \& Zhang (1996) reported that the spectra of V711 Tau obtained by them in the phase interval $0.855-0.962$ exhibit strong absorption features centred about the expected $\mathrm{H} \alpha$ absorption line of the secondary. Four of the spectra obtained by us fall in the phase interval $0.838-0.883$. Out of these only 
that obtained on 22 January 2009 (JD 2 454 854.1573) at $0.845 p$ exhibits an absorption feature close to the expected position of the $\mathrm{H} \alpha$ line of the secondary; that position also coincides with the position of the water vapour line at $6564.206 \AA$. Most likely, the absorption feature seen by Zhai \& Zhang (1996) in the phase interval $0.855-0.962 p$ is also this line. An inspection of the $\mathrm{H} \alpha$ profiles presented by them shows that the absorption feature is prominent only in the spectra taken at $0.855 \mathrm{p}$ and $0.902 p$, which are close to the phase where we also detected an absorption feature. We note that their observations were performed during 13-19 January 1993, and the optimal combination of the orbital velocity of the Earth and the heliocentric velocity of the secondary made the expected position of its $\mathrm{H} \alpha$ line at these phases close to that of the water line. The spectrum obtained by Gondoin (1986) at 0.94p on 22 September 1983 (JD 2445600.822 ) does not show any strong absorption feature at the expected position of the $\mathrm{H} \alpha$ line of the secondary. Zhai \& Zhang (1996) had proposed a mass transfer model to explain the structure of $\mathrm{H} \alpha$ emission, based partly on the phasedependent appearance of the $\mathrm{H} \alpha$ absorption line of the secondary. Additional high resolution observations of V711 Tau are needed to determine the exact contribution of the secondary component to the observed $\mathrm{H} \alpha$ profile.

There is no indication of the $\mathrm{H} \alpha$ line of the secondary in either emission or absorption in the spectra obtained by us. It is known to exhibit strong $\mathrm{Ca}$ II $\mathrm{H}$ and $\mathrm{K}$ emission (Bopp \& Fekel 1976; Montes et al. 1995) and probably displays a filled-in $\mathrm{H} \alpha$ line.

The base-line width of the $\mathrm{H} \alpha$ emission outside the flare, which is a measure of the width of the broad emission component found by Montes et al. (1997), does not show any clear evidence of a modulation that is anti-correlated with the light variation; this is inconsistent with the suggestion by Lanzafame et al. (2000) that the broad emission component originates in more localised active regions. However, the base-line width seems to exhibit a larger fluctuation during the phases of light curve minimum than during the phases of light curve maximum. The larger base-line widths during the phases of minimum light are, probably, caused by the more frequent low intensity flares. The equivalent widths of $\mathrm{H} \alpha$ emission also do not show any clear modulation with the orbital phase. But, the spread in the variation in the equivalent width seems to be anti-correlated with the light variation.

\section{Conclusions}

By analysing the available times of maximum of radial velocity of the K-type component during 1976-2009, we have found that the orbital period of V711 Tau has a near-sinusoidal modulation with a $36.3 \pm 1.9 \mathrm{yr}$ period. The orbital period and initial epoch of the star so far used in the literature need slight corrections. The near-constancy of the O-C during 1976-90 and its subsequent steep fall reported by Frasca \& Lanza (2005) and Lanza et al. (2006) are a result of the slightly longer orbital period used by them in their analysis. An analysis of the new photometry and those in the literature shows that the spot activity in V711 Tau varies cyclically with an average period of $14.1 \pm 0.3 \mathrm{yr}$. The maximum of the spot activity cycle appears to have a long-term modulation. It is found that even during the times of minimum spot activity, a substantial fraction, probably, larger than $20 \%$, of the surface of the active star is covered by spots.

We have attributed the near-constancy of the composite $(B-V)$ colour of V711 Tau as the active star becomes fainter to the increased fractional contribution by the hotter component to the total light in the blue spectral region. We have found that the difference in the $V$ magnitudes of the components of V711 Tau estimated using their radii quoted in the literature and Barnes-Evans relation to be around 1 mag more than that suggested by their spectra. It appears that the radius of the secondary component derived from its $v$ sin $i$ and used in several spot modelling studies in the literature is grossly underestimated. There is a likelihood that the secondary in V711 Tau does not rotate synchronously with its orbital motion, and (or) its equatorial and orbital planes are not coplanar. The situation is probably similar in the case of the RS CVn binary UX Ari. Detailed spectroscopic analyses of these and similar binaries are needed to determine the ratio of the radii of the components more accurately before any definite conclusion can be made.

We do not find the expected decrease in the equivalent widths of $6430.84 \AA$ and $6439.07 \AA$ lines of the active star in V711 Tau from those of a single star of similar spectral type because of the presence of the continuum flux from its companion, indicating that the cool spotted regions contribute significantly to the observed absorption line strengths.

The orbital modulation in V711 Tau seems to be wellcorrelated with the spot activity of the cool component, indicating that physical processes associated with its magnetic activity are definitely responsible for the orbital period variation. The present results are qualitatively consistent with the torsional oscillation model discussed by Lanza \& Rodono (2004). Further simultaneous radial velocity measurements and photometry are needed to determine more accurately the ratio of the periods of spot activity cycle and orbital period modulation.

There is a suggestion from the existing photometry of V711 Tau that there is a longitudinal asymmetry in spot activity that is fixed in the orbital frame of reference. Acquiring photometry of V711 Tau during the forthcoming minimum of spot activity cycle will be very important in this connection. The evidence of a longitudinal asymmetry that is fixed in the orbital frame of reference is stronger in the case of UX Ari. It is extremely essential to exclude the possibility that the asymmetries observed in V711 Tau and also in UX Ari are artifacts produced by the existing data samples because these asymmetries, if found to be real, would mean that the role of binarity is not just limited to the spinning up of the active star by locking its rotation with the orbital motion; it would also mean that the binarity plays a role in either completely suppressing the surface differential rotation of the active star or creating a phase-locked surface spot activity, resulting in preferred longitudes where spots form and disintegrate more often.

The variation in the equivalent width and base-line width of $\mathrm{H} \alpha$ emission in V711 Tau are not correlated with the light modulation; but, if we exclude the observation during an intense flare activity, there is an indication that both show larger fluctuations during light curve minimum than during light curve maximum. When the present spectroscopic observations were made, the amplitude of variation in $V$ band was only $\sim 0.05 \mathrm{mag}$, which is indicative of a low level of asymmetry in the distribution of spots on the visible surface of the active star; therefore, it is difficult to rule out conclusively the absence of any clear modulation in the equivalent width and base-line width of $\mathrm{H} \alpha$ emission in V711 Tau. Spectroscopic observations when V711 Tau exhibits larger amplitudes of light variation are needed.

Acknowledgements. We thank Dr Padmakar Parihar for allowing us to use the spectra of V711 Tau taken during 2006, the staff at VBO for their help at the telescope, and Dr A. F. Lanza for sending us promptly the individual observations of V711 Tau. We also thank Prof. Petr Hadrava for valuable comments. 


\section{References}

Alekseev, I. Yu. 2005, Astrophys., 48, 20

Applegate, J. H. 1992, ApJ, 385, 621

Bagnulo, S., Cabanac, R., Jehin, E., Ledoux, C., \& Melo, C. 2003, The UVES Paranal Observatory Project, http://www.sc.eso.org/santiago/ uvespop/field_stars_uptonow.html

Barnes, T. G., \& Evans, D. S. 1976, MNRAS, 174, 489

Berdyugina, S. V., \& Henry, G. W. 2007, ApJ, 659, L157

Bopp, B. W. 1983, in Activity in Red-dwarf Stars, ed. P. B. Byrne, \& M. Rodono (Dordrecht: Reidel), Proc. IAU Coll., 71

Bopp, B. W., \& Fekel, F. 1976, AJ, 81, 771

Carlos, R. C., \& Popper, D. M. 1971, PASP, 83, 504

Dean, J. F. 1979, MNASS Africa, 38, 79

Donati, J.-F. 1999, MNRAS, 302, 457

Donati, J.-F., Brown, S. F., Semel, M., et al. 1992, AA, 265, 682

Donati, J.-F., Cameron, A. C., Semel, M., et al. 2003, MNRAS, 345, 1145

Drilling, J. S., \& Landolt, A. U. 2000, in Allen's Astrophysical Quantities, ed. A. N. Cox

Duemmler, R., \& Aarum, V. 2001, A\&A, 370, 974

Eggen, O. J. 1966, RGOB, 120, 333

Eggen, O. J. 1978, IBVS, 1426

Fekel, F. C. 1983, ApJ, 268, 274

Frasca, A., \& Lanza, A. F. 2005, A\&A, 429, 309

Fraquelli, D. A. 1982, ApJ, 254, L41

Garcia-Alvarez, D., Barnes, J. R., Collier Cameron, A., et al. 2003, A\&A, 402, 1073

Gondoin, P. 1986, A\&A, 160, 73

Hall, D. S. 1990, in Active close binaries, ed. C. Ibanoglu (Dordrecht: Kluwer Academic), 95

Hatzes, A. P., Vogt, S. S., Ramseyer, T. F., \& Misch, A. 1996, ApJ, 469, 808

Heintz, W. D. 1978, Double Stars (Dordrecht, Holland: D. Reidel Publishing Company)

Henry, G. W., Eaton, J. A., \& Hamer, J. 1995, ApJS, 97, 513

Hoffleit, D., \& Jaschek, C. 1982, The Bright Star Catalogue, Yale University Observatory, USA
Landis, H. J., \& Hall, D. S. 1976, IBVS, 111

Lanza, A. F. 2005, MNRAS, 364, 238

Lanza, A. F., \& Rodono, M. 2004, AN, 325, 393

Lanza, A. F., Rodono, M., \& Rosner, R. 1998, MNRAS, 296, 893

Lanza, A. F., Piluso, N., Rodono, M., Messina, S., \& Cutispoto, G. 2006, A\&A, 455,595

Lanzafame, A. C., Busa, I., \& Rodono, M. 2000, A\&A, 362, 683

Mekkaden, M. V., \& Raveendran, A. V. 1998, A\&A, 338, 1031

Mekkaden, M. V., Raveendran, A. V., \& Mohin, S. 1982, JApA, 3, 27

Mohin, S., \& Raveendran, A. V. 1993, A\&AS, 100, 331

Montes, D., Fernandez-Figueroa, M. J., De Castro, E., \& Cornide, M. 1995, A\&AS, 109, 135

Montes, D., Fernandez-Figueroa, M. J., De Castro, E., \& Sanz-Forcada, J. 1997, A\&AS, 125, 263

Nations, H. L., \& Ramsey, L. W. 1986, AJ, 92, 1403

Parthasarathy, M., Raveendran, A. V., \& Mekkaden, M. V. 1981, Astrophys. Sp. Sci., 74, 87

Petit, P., Donati, J.-F., Wade, G. A., et al. 2004, MNRAS, 348, 1175

Popper, D. M. 1978, AJ, 83, 1522

Rao, N. K., Sriram, S., Jayakumar, K., \& Gabriel, F. 2005, JApA, 26, 331

Rosario, M. J., Raveendran, A. V., \& Mekkaden, M. V. 2007, A\&A, 474, L41

Rosario, M. J., Mekkaden, M. V., \& Raveendran, A. V. 2008, IBVS, 5836

Rosario, M. J., Heckert, P. A., Mekkaden, M. V., \& Raveendran, A. V. 2009, MNRAS, 394, 872

Scharlemann, E. T. 1982, ApJ, 253, 298

Stawikowski, A., \& Glebocki, R. 1994, Acta Astr., 44, 393

Tas, G., Evren, S., \& Ibanoglu, C. 1999, A\&A, 349, 546

Ulvas, V. A., \& Engvold, O. 2003, A\&A, 399, L11

Ulvas, V. A., \& Henry, G. W. 2005, AN, 326, 292

Vogt, S. S., Hatzes, A. P., Misch, A. A., \& Kurster, M. 1999, ApJS, 121, 547

Wacker, S. W., Guinan, E. F., McCook, G. P., Lochner, J. C., \& Paczkowski, B. G. 1986, IBVS, 2920

Wilson, R. E. 1953, The General Catalogue of Stellar Radial Velocities

Zhai, D. S., \& Zhang, X. B. 1996, A\&A, 309, 530 